\title{
FAECAL CARRIAGE OF MULTI-DRUG RESISTANCE MICROORGANISMS IN HEALTHY INFANTS
}

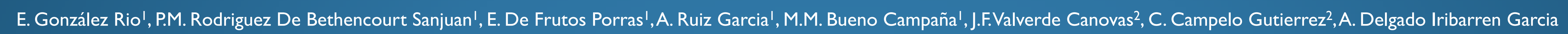
Campero', G.M. Botija Arcos'. 'Paediatric, Hospital Universitario Fundación Alcorcón, Madrid, Spain. 2 Microbiology, Hospital Universitario Fundación Alcorcón, Madrid, Spain.

BACKROUND There is an alarming increase of multi-drug resistance microorganisms (MDRM) in our environment.

Different studies have analyzed the MDRM prevalence on healthy children, obtaining heterogeneous results depending on the regional area and the age.

The aim of our study is to analyze prevalence of MDRM faecal colonization and evaluate associated risk factors in infants up to 12 months old

METHODS Prospective follow-up study. A cohort of healthy newborn children

$\checkmark$ Gestational age

$\checkmark$ Newborn weight

$\checkmark$ Maternal GBS colonization

$\checkmark$ Duration of membrane's rupture

$\checkmark$ Perinatal antibiotic

$\checkmark$ NICU admission

\begin{tabular}{|c|c|c|c|}
\hline \multicolumn{2}{|c|}{ Newborns } & th & \\
\hline \multirow{2}{*}{\multicolumn{2}{|c|}{$\begin{array}{l}\text { Perinatal history } \\
\text { Neonatal history }\end{array}$}} & \multicolumn{2}{|c|}{ Stool sample } \\
\hline & & \multicolumn{2}{|c|}{ Cuestionarie } \\
\hline \multicolumn{4}{|c|}{$.4 \%$ female) } \\
\hline ks & \multicolumn{2}{|c|}{ Vaginal deliveries } & $88 \%$ \\
\hline$(-40,14)$ & \multirow{2}{*}{\multicolumn{2}{|c|}{$\begin{array}{c}\text { Maternal Group B } \\
\text { streptococcus } \\
\text { colonization }\end{array}$}} & \multirow[b]{2}{*}{$12.6 \%$} \\
\hline-3446$)$ & & & \\
\hline \multirow{2}{*}{$\begin{array}{l}\text { rs } \\
.11,1)\end{array}$} & \multicolumn{2}{|c|}{ Perinatal antibiotics } & $15 \%$ \\
\hline & \multicolumn{2}{|c|}{ Hospital's admission } & $13.1 \%$ \\
\hline
\end{tabular}

6 months of age

Stool sample

Cuestionarie $\checkmark$ weight

$\checkmark$ history of gastroenteritis,

$\checkmark$ hospital's admissions,

$\checkmark$ antibiotic intake

$\checkmark$ antacids intake

$\checkmark$ day-care centre assistance

$\checkmark$ breast-feeding or infant

formula

$\checkmark$ co-habitant and housepet
RESULTS 178 patientes recruited (51.4\% female)

\begin{tabular}{|c|c|}
\hline Gestational age & $\begin{array}{c}\text { 39,7 weeks } \\
\text { (IC25-75p: 38,7I-40, I4) }\end{array}$ \\
\hline Newborn weight & $\begin{array}{c}\text { 3I II } \mathbf{g r} . \\
\text { (IC25-75:29|2-3446) }\end{array}$ \\
\hline $\begin{array}{c}\text { Duration of } \\
\text { membrane's rupture }\end{array}$ & $\begin{array}{c}\mathbf{8 , 5} \text { hours } \\
\text { (IC25-75: I,5-II,I) }\end{array}$ \\
\hline
\end{tabular}

There were no significant differences in analyzed's variables.

Prevalence of MDRM in children whose mothers were colonized by Group B streptococcus at birth (I9\% vs $5.9 \%$, $p=0.06)$

\begin{tabular}{|c|c|c|}
\cline { 2 - 3 } \multicolumn{1}{c|}{} & $\begin{array}{c}\text { Sample } \\
\text { analyzed }\end{array}$ & $\begin{array}{c}\text { Number of children } \\
\text { colonized by MDRM }\end{array}$ \\
\hline $\begin{array}{c}\mathrm{I}^{\circ} \text { month } \\
\text { of age }\end{array}$ & $\mathbf{1 7 4}$ & $\mathbf{I}(\mathbf{0 . 6 \% )}$ \\
\hline $\begin{array}{c}6^{\circ} \text { month } \\
\text { of age }\end{array}$ & 157 & $\mathbf{1 2 ( 7 . 6 \% )}$ \\
\hline
\end{tabular}

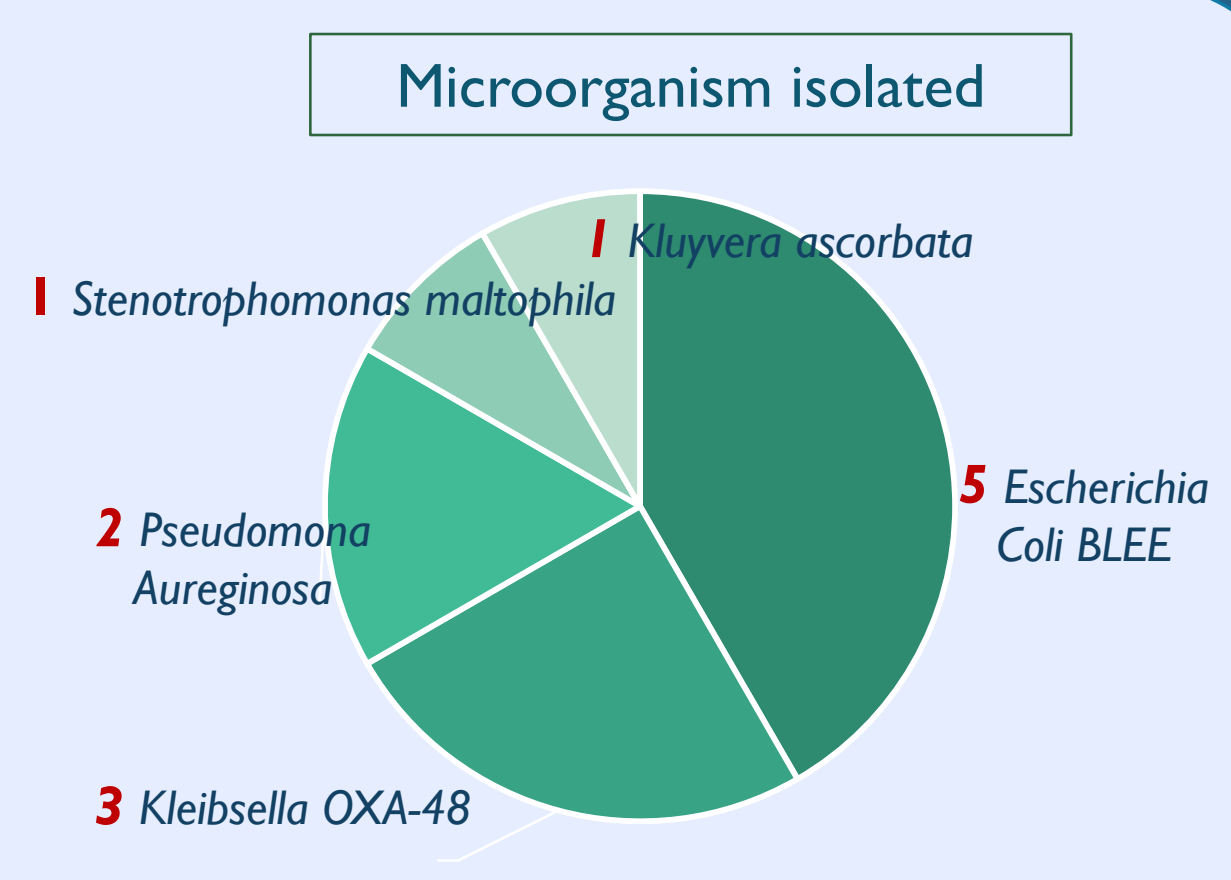

CONCLUSIONS MDRM faecal colonization in healthy children in our hospital (pending of complete sample) seems to be high, similar to those reported in developed countries, underlining the importance of this population as MDRM's reservoir 